\title{
RECENT RESULTS ON THE HELICITY STRUCTURE OF THE NUCLEON FROM HERMES
}

\author{
MARC BECKMANN \\ on behalf of the HERMES Collaboration \\ Deutsches Elektronen-Synchrotron DESY, D-22603 Hamburg, Germany \\ E-mail: marc.beckmann@desy.de
}

\begin{abstract}
The HERMES experiment has measured double spin asymmetries of inclusive and semi-inclusive cross sections for the production of charged hadrons in deepinelastic scattering of polarised positrons on polarised hydrogen and deuterium targets, in the kinematic range $0.023<x<0.6$, and $1 \mathrm{GeV}^{2}<Q^{2}<15 \mathrm{GeV}^{2}$. For the data taken on the deuterium target, a RICH detector provides the complete identification of charged pions and kaons. From the inclusive measurements on deuterium, the polarised structure function $g_{1}^{d}$ has been extracted with high precision. Together with the semi-inclusive measurements, quark polarisations are extracted separately for the $u, \bar{u}, d, \bar{d}$, and $(s+\bar{s})$ flavours in a LO QCD analysis. Furthermore, a possible breaking of flavour symmetry in the polarised light sea, $\Delta \bar{u}-\Delta \bar{d}$, was measured for the first time and found to be consistent with zero within the experimental precision.
\end{abstract}

\section{Formalism of the Analysis}

Cross section asymmetries measured in doubly polarised deep-inelastic scattering (DIS) provide a useful tool to probe the helicity structure of the nucleon. Under the assumption of a vanishing spin structure function $g_{2}=0$, the experimentally measured asymmetry $A_{\|}$is related to the asymmetry $A_{1}$ in the virtual photon - nucleon reference frame by

$$
\frac{A_{\|}}{D(1+\eta \gamma)} \simeq A_{1} \simeq \frac{g_{1}}{F_{1}}
$$

Here, $D$ is the depolarisation factor of the virtual photon, $g_{1}$ and $F_{1}$ are the polarised and the unpolarised nucleon structure functions, respectively, and $\eta$ and $\gamma=\sqrt{Q^{2} / \nu^{2}}$ are kinematic factors. Here, $\nu$ is the energy of the virtual photon, $-Q^{2}$ is its four-momentum squared, and $x=Q^{2} / 2 M \nu$, where $M$ is the nucleon rest mass. Contributions from $g_{2}$, found to be very small in measurements at SLAC [1], are omitted. A residual effect of the non-zero value of $g_{2}$ on the extracted quark polarisations has been included 
in the systematic uncertainty.

In leading order (LO) QCD the relation $g_{1}\left(x, Q^{2}\right)=\sum_{q} e_{q}^{2} \Delta q\left(x, Q^{2}\right)$ applies, where $\Delta q\left(x, Q^{2}\right) \equiv q^{+}\left(x, Q^{2}\right)-q^{-}\left(x, Q^{2}\right)$ are the helicity quark distributions in the nucleon. Information on the charge squared weighted sum over the quark helicity distributions can hence be obtained based on measurements of $g_{1}$ according to Eq. (1). Here, $q^{+(-)}\left(x, Q^{2}\right)$ is the number density of quarks with spin aligned parallel (anti-parallel) to the nucleon's spin.

The observation of coincident hadrons from the current fragmentation region in semi-inclusive deep-inelastic scattering (SIDIS) processes provides access to the flavour of the struck quark. Assuming factorisation, the SIDIS cross section asymmetry $A_{1}^{h}$ can be written in LO QCD as

$$
A_{1}^{h}\left(x, Q^{2}\right) \stackrel{g_{2}=0}{\simeq} \frac{1+R\left(x, Q^{2}\right)}{1+\gamma^{2}} \times \frac{\sum_{q} e_{q}^{2} \Delta q\left(x, Q^{2}\right) \int_{z_{\min }}^{z_{\max }} D_{q}^{h}\left(Q^{2}, z\right) \mathrm{d} z}{\sum_{q} e_{q}^{2} q\left(x, Q^{2}\right) \int_{z_{\min }}^{z_{\max }} D_{q}^{h}\left(Q^{2}, z\right) \mathrm{d} z} .
$$

Here, $q\left(x, Q^{2}\right)$ denote the unpolarised parton densities (PDFs) and $R\left(x, Q^{2}\right)=\sigma_{L} / \sigma_{T}$ is the photo-absorption cross section ratio for longitudinal and transverse virtual photons. In this analysis, the fragmentation functions $D_{q}^{h}\left(Q^{2}, z\right)$ are integrated over the range $z_{\min }=0.2$ to $z_{\max }=0.8$ in $z=E_{h} / \nu$, which is the fraction of the virtual photon's energy carried by the hadron of type $h$. The upper cut on $z \leq 0.8$ eliminates exclusive events from the sample.

For a set of measured inclusive and semi-inclusive asymmetries on different targets, after integrating over the range in $Q^{2}$ in each $x$-bin, Eq. (2) can be rewritten in matrix form

$$
\overrightarrow{\mathrm{A}}(x)=P(x) \overrightarrow{\mathrm{Q}}(x) .
$$

The vectors $\overrightarrow{\mathrm{A}}(x)$ and $\overrightarrow{\mathrm{Q}}(x)$ contain the measured asymmetries and the polarisations $\Delta q / q$ of the different quark flavours to be extracted, respectively. The elements of the matrix $P(x)$ depend on the fragmentation functions, the PDFs, the cross section ratio $R\left(x, Q^{2}\right)$, and on the relative fluxes of hadrons originating from the different nucleons in case of deuterium. $P(x)$ also contains the influence from the limited acceptance of the spectrometer. The fragmentation was modelled in the LUND string model implemented in the JETSET 7.4 [2] package. In order to obtain a reliable description of the fragmentation at HERMES energies, the LUND string breaking parameters have been tuned to fit the hadron multiplicities measured at HERMES. As an estimate of the associated systematic uncertainties, the analysis was also carried out with an alternative fit to the measured multiplicities and with a default LUND parameter setting for the PETRA $e^{+} e^{-}$collider. The result- 

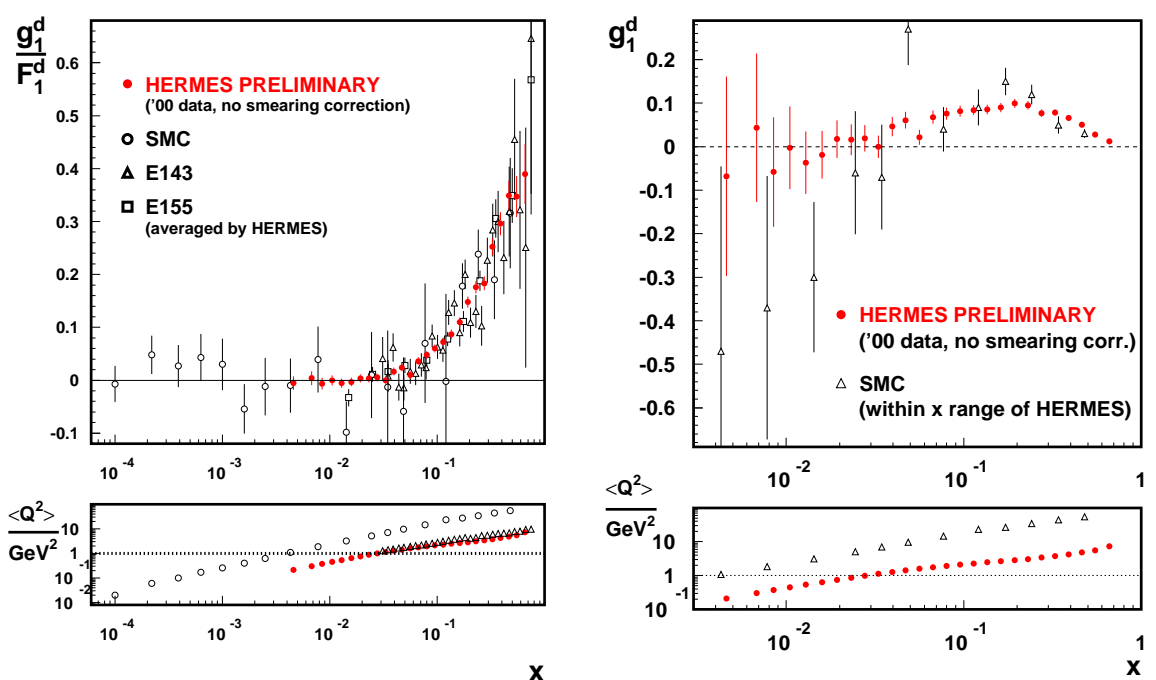

Figure 1. Preliminary HERMES results for $g_{1}^{d} / F_{1}^{d}$ and $g_{1}^{d}$ as a function of $x$.

ing variations of the extracted quark polarisations were added in quadrature to the other sources of systematic uncertainties. For the unpolarised PDFs the parameterisations of Ref. [3] (CTEQ5LO) were used.

The flavour decomposition of the quark polarisations was obtained by solving Eq. (3) for the vector $\overrightarrow{\mathrm{Q}}(x)=\left(\frac{\Delta u}{u}, \frac{\Delta \bar{u}}{\bar{u}}, \frac{\Delta d}{d}, \frac{\Delta \bar{d}}{d}, \frac{\Delta s}{s} \equiv \frac{\Delta \bar{s}}{\bar{s}}\right)$. In contrast to earlier analyses $[4,5]$, the only remaining symmetry assumption is $\Delta s(x) / s(x)=\Delta \bar{s}(x) / \bar{s}(x)$.

\section{Experimental Results}

The HERMES experiment [6] uses the longitudinally polarised $27.6 \mathrm{GeV}$ positron beam of the HERA storage ring at DESY, incident on longitudinally polarised pure atomic gas targets of ${ }^{3} \mathrm{He}$, hydrogen or deuterium. A large acceptance forward spectrometer with good particle identification detects the scattered beam positrons together with coincident final state hadrons. A RICH detector installed in 1998 provides the identification of pions, kaons and protons over a wide momentum range for the data on the polarised deuterium target taken since then.

\subsection{Inclusive Measurements}

Fig. 1 shows the preliminary HERMES data on the structure function ratio $g_{1}^{d} / F_{1}^{d}$ and on the polarised structure function $g_{1}^{d}$. These very precise results are based on 10 million events taken on a polarised deuterium target 

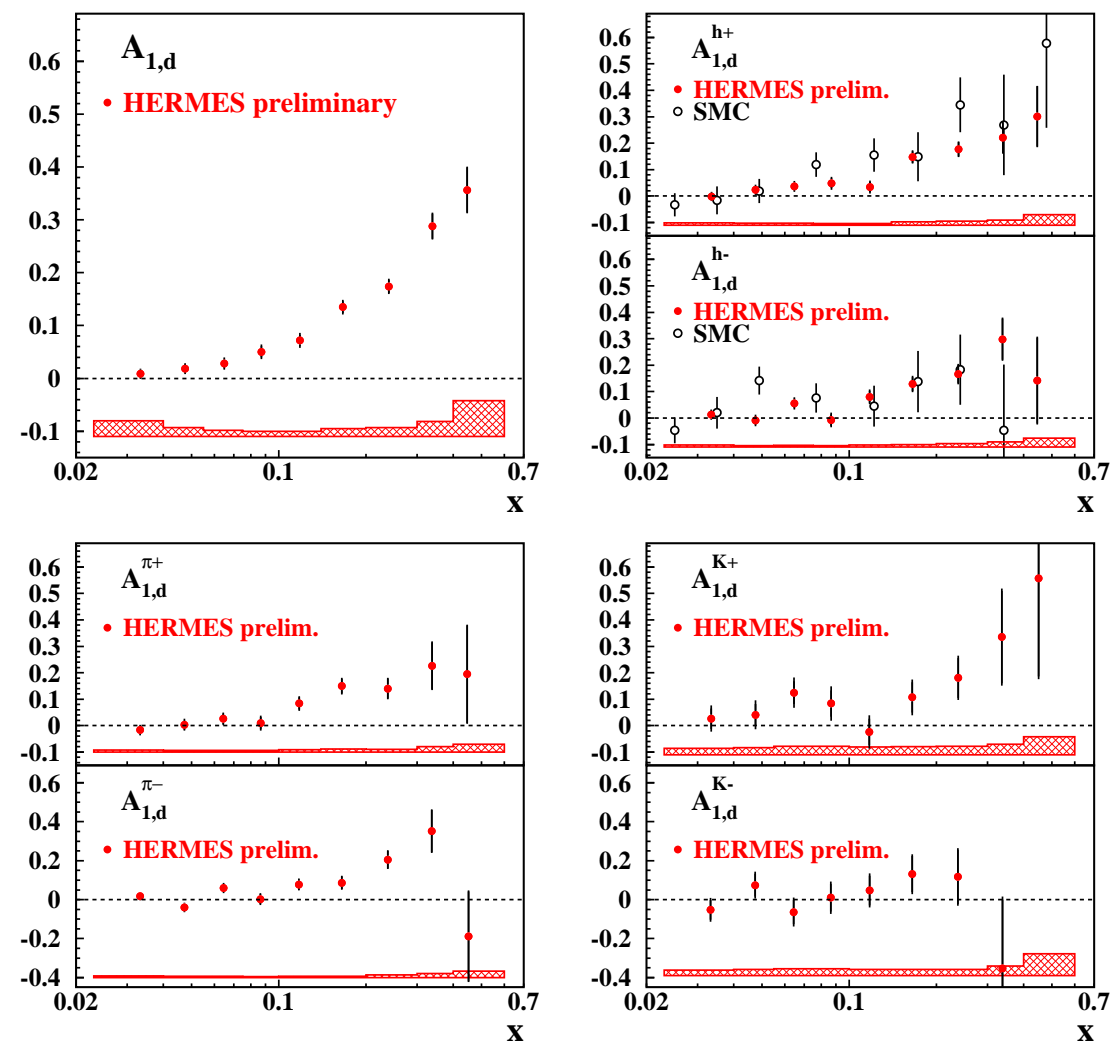

Figure 2. Preliminary HERMES results on inclusive and semi-inclusive DIS cross section asymmetries $A_{1, d}(x)$ on a polarised deuterium target.

in the kinematic range $Q^{2}>0.1 \mathrm{GeV}^{2}$ and $W^{2}>3.24 \mathrm{GeV}^{2}$. Despite the differences in the average values of $\left\langle Q^{2}\right\rangle$ per bin, one observes a good consistency of the HERMES data on $g_{1} / F_{1}$ with an earlier measurement of SMC at larger values of $\left\langle Q^{2}\right\rangle$ and measurements at SLAC, taken at similar average values of $Q^{2}$. This close agreement is a clear indication for very similar $Q^{2}$ evolutions for both the polarised and the unpolarised structure functions over the range in $Q^{2}$ covered by experiments.

\subsection{Semi-Inclusive Measurements}

The preliminary data presented here are based on 1.8 (6.5) million DIS events taken on a hydrogen (deuterium) target in the kinematic range $Q^{2}>1 \mathrm{GeV}^{2}$ and $W^{2}>10 \mathrm{GeV}^{2}$. For the hydrogen data set, final state pions could be identified using the information from a threshold Cerenkov counter, while for the deuterium data set the RICH detector provided kaon 

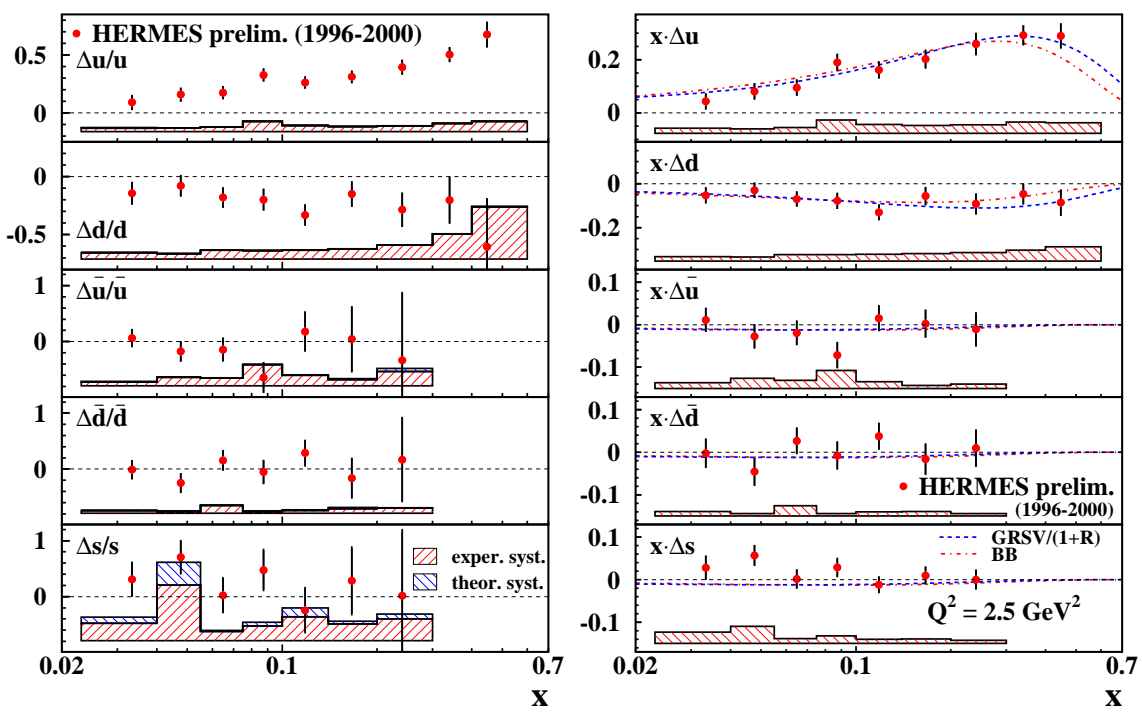

Figure 3. HERMES preliminary results on the polarisation of the $u, \bar{u}, d, \bar{d}$, and $s+\bar{s}$ quark flavours versus $x$ (left panel). The right panel shows the results on the corresponding polarised quark distributions at a common scale of $Q^{2}=2.5 \mathrm{GeV}^{2}$. The dashed and the dotted-dashed lines are parameterisations from Refs. [9, 10]. The error bars are statistical, while the shaded bands indicate the systematic uncertainties of the results. The contribution labelled "theor. syst." in the left panel gives the uncertainty due to with the use of two different unpolarised parton densities [3, 11].

identification as well. The preliminary results for the inclusive and semiinclusive asymmetries on the deuterium target are shown in Fig. 2.

Together with a set of inclusive and semi-inclusive asymmetries on a polarised proton target, the polarisation of five quark flavours was extracted according to Eq. (3). For the sea quark flavours, at values of $x>0.3$ the polarisation was set to zero. The resulting small influence on the up and down quark polarisations was included in the systematic uncertainties. Fig. 3 shows the obtained quark polarisations as well as the polarised quark distributions. The polarisation of the up quarks is positive everywhere in the measured range and increases with $x$ up to 0.7 at $x=0.47$. The down quark polarisation ranges between -0.1 and -0.5 , almost independently of $x$. For the light sea quarks the polarisation is compatible with zero, while for the strange quarks a slightly positive polarisation is favoured within the measured range in contrast to results based on inclusive data alone (e.g. [9, 10]). However, within their total uncertainty also the polarisation of the strange quarks is zero.

Fig. 4 shows a first measurement of the difference of the polarised light sea flavours $\Delta \bar{u}(x)-\Delta \bar{d}(x)$ together with predictions of a broken $\mathrm{SU}(2)_{f}$ 


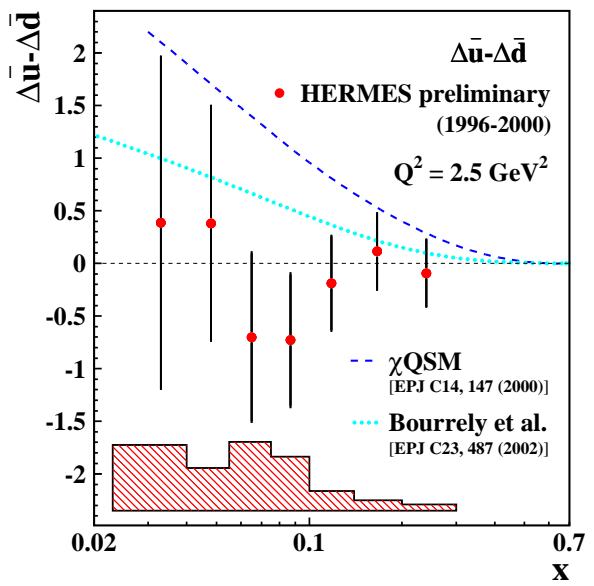

Figure 4. HERMES preliminary result on the difference of the polarised light sea $\Delta \bar{u}-$ $\Delta \bar{d}$ as a function of $x$ at a scale of $Q^{2}=2.5 \mathrm{GeV}^{2}$.

symmetry from Refs. $[7,8]$. The data are consistent with an unbroken $\mathrm{SU}(2)_{f}$ symmetry in the light polarised sea.

\section{Conclusions}

HERMES has collected large statistics of inclusive and semi-inclusive DIS data on pure targets of polarised hydrogen and deuterium. From the inclusive data precise results on the polarised structure function $g_{1}^{d}$ were extracted. Based on a LO QCD analysis of semi-inclusive data taken on both target nuclei, polarised parton densities for $u, \bar{u}, d, \bar{d}$, and $(s+\bar{s})$ flavours have been extracted as a function of $x$ in the range $0.023<x<0.6$. A first measurement of the flavour symmetry in the polarised light sea $\Delta \bar{u}-\Delta \bar{d}$ is compatible with the $\mathrm{SU}(2)_{f}$ assumption.

\section{References}

1. Anthony, P.L. et al., hep-ex/0204028.

2. Sjöstrand, T., Comp. Phys. Comm. 82, 74 (1994).

3. Lai, H.L. et al., Eur. Phys. J. C12, 375 (2000).

4. Adeva, B. et al., Phys. Lett. B 420, 180 (1998).

5. Ackerstaff, K. et al., Phys. Lett. B 464, 123 (1999).

6. Ackerstaff, K. et al., Nucl. Instr. Meth. A417, 230 (1998).

7. Dressler, B. et al., Eur. Phys. J. C14, 147 (2000).

8. Bourrely, C. et al., Eur. Phys. J. C23, 487 (2002).

9. Glück, M. et al., Phys. Rev. D 63, 094005 (2001).

10. Blümlein, J., Böttcher, H., hep-ph/0203155, Nucl. Phys. B, (2002) in press.

11. Glück, M. et al., Eur. Phys. J. C5, 461 (1998). 